\title{
Adaptive Dual Conjudate Gradient Projection Algorithm for Compressed Sensing Image Reconstruction
}

\author{
Yan Haixia \\ Electronic Department \\ ,JiLin University \\ Changchun 130012,China \\ yanhx@jlu.edu.cn
}

\author{
Liu Yanjun \\ Changchun Institute of Optics, Fine Mechanics and Physics \\ the Chinese Academy of Sciences \\ Changchun 130012,China \\ liuyanjun@ciomp.ac.cn
}

\begin{abstract}
In order to improve the quality of noise signals reconstruction method, an algorithm of adaptive dual gradient projection for sparse reconstruction of compressed sensing theory is proposed. In ADGPSR algorithm, the pursuit direction is updated in two conjudate directions, the better original signals estimated value is computed by conjudate coefficient. Thus the reconstruction quality is improved. Experiment results show that, compared with the GPSR algorithm, the ADGPSR algorithm improves the signals reconstruction accuracy, improves PSNR of reconstruction signals, and exhibits higher robustness under different noise intensities.
\end{abstract}

Keywords- signal processing, gradient projection, compressed sensing, image reconstruction

\section{INTRODUCTION}

The Nyquist sampling theorem of information theory is the measurement rules of signal sample, which is proposed by the American Electrical Engineer H. Nyquist. In 1928 the founder of information theory C.E. Shannon proves the Shannon sample theory and cites it as a theorem ${ }^{[1,2]}$. The Shannon sample theory occupy all areas of signal acquisition, image acquisition, processing, storage, transmission, etc. The Shannon sample theory works in the way of that the sampling rate not less than two times the highest frequency ${ }^{[3-}$ 5].

With the development of information technology, there are two question of the Shannon sample theory. The first question is that the data acquisition and processing is difficult in some conditions, such as the ultra-wideband communication system, UWB signal processing, $\mathrm{THz}$ imaging, nuclear magnetic resonance, space exploration, and so on. Second question is that the data storage and transmission $n$ a significant degree of waste of resources ${ }^{[6-8]}$.

In recent years, the compressed sensing theory proposed by Candes, Romberg, et al (Compressed sensing) to overcome the shortcoming of Nyquist sampling theory ${ }^{[9,10]}$, the basic idea is that, sampling the signals with a lower sample rate, rather than the higher sample rate, then compressing the sampled signal. The basic of this theory is the sparse signals can be compressed. The theory can use far less than the Nyquist sampling frequency of data sampling, and still be able to accurately restore the signal ${ }^{[11,12]}$.The outstanding advantages of compressed sensing theory are that, for those signals with a sparse representation, the theory combine separately data acquisition with data compression into one process ${ }^{[13,14]}$. Thus the signal acquisition on the acquisition of equipment is reduced, especially for high resolution acquisition signals, the signal acquisition time and storage space greatly have been reduced. the cost of signal acquisition and processing are saved ${ }^{[15,16]}$.

Point to the reconstruction quality of GPSR algorithm, we propose an ADGPSR algorithm (adaptive dual gradient projection for sparse reconstruction). In ADGPSR when computing the gradient projection directions, we compute two conjugate searching directions. The signals reconstruction quality of ADGPSR is higher than GPSR algorithm, At the same time, the ADGPSR algorithm improves the SPNR of reconstruction image and exhibits higher robustness under different noise intensities.

\section{COMPRESSED SENSING THEORY AND IMAGE RECONSTRUCIONT METHOD}

\section{A Compressed sensing theory}

In compressed sensing theory, the signal must be sparse, or can be represented as sparse by some transforms. In general, the signals are not sparse, after a certain transformation (such as wavelet transform), the signals can be considered to be sparse, for example, after the wavelet transform, the transform results which contain $\mathrm{K}$ major results, and other N-K results are set to zero. Assume that the original signal to be processed for $f \in R^{N}$, it's sparse basis is matrix $\Psi$, in this way the signal $f$ is sparse on the base $\Psi$, this process can be expressed as formula (1):

$$
f=\Psi x
$$

where $X$ is the decomposition of the system, it has a sparse features as formula (2):

$$
\|x\|_{0} \leq k
$$

The symbol $\|x\|_{0}$ is the norm of signal $l_{0}$, a number of non-zero value vector. After the signal is sparse representation, the random measurement can be complete by an observation matrix as formula (3):

$$
y=\Phi f=\Phi \Psi x=A x
$$

where $y$ is the measurement vector, $y \in R^{M}$, $M<<N$,because the signal has a sparse, the decoding 
process of the above-mentioned problems can be solved by the following formula:

$$
\min \|x\|_{0} \text { s.t. } y=A x
$$

When the coefficient vectors are get, the signal $f$ can be restored by $f=\Psi x$.

However, formula (4) is a NP-hard problem, it can't be solved within a limited time. One of the most important contribution of the compressed sensing theory is that the question of norm $l_{0}$ and be equal to question norm $l_{1}$, when the signals are sparse and the observation matrix satisfy certain conditions.

$$
\min \|x\|_{1} \text { s.t. } y=A x
$$

The symbol $\|x\|_{1}$ indicates that norm of $l_{1}$, the absolute value of the vector. Solving problems of the norm is a convex optimization problem, can be resolved by linear programming.

\section{$B$ ADGPSR reconstruction method}

In the actual measurement process noise will be introduced inevitably, the model can be expressed as:

$$
y=O x+n
$$

Where $\mathrm{n}$ is the measurement noise vector. In this case, the signal reconstruction process can be expressed as:

$$
\min _{x}\|x\|_{1} \text { subject }\|y-A x\|_{2}^{2} \leq \varepsilon
$$

The algorithm, developed from the damped Newton method, for an unconstrained optimization of smooth nonlinear function $\mathrm{F}$, it is every step of the formula

$$
\delta^{(k)}=-H_{k}^{-1} F\left(z^{k}\right)
$$

Because the $H_{k}$ can not be calculated, need to figure out:

$$
\nabla F\left(z^{k}\right)-\nabla F\left(z^{k-1}\right) \approx \eta^{k}\left[z^{k}-z^{k-1}\right]
$$

Approximation algorithm of $H_{k}$ is not satisfied, increase of algorithm iterations.

$$
\min c^{T} z+\frac{1}{2} z^{T} B z=F(z)
$$

where , $\quad z=\left[\begin{array}{l}u \\ v\end{array}\right], \quad b=A^{T} y, c=\tau 1_{2 n}+\left[\begin{array}{l}-b \\ b\end{array}\right]$, $B=\left[\begin{array}{c}A^{T} A,-A^{T} A \\ -A^{T} A, A^{T} A\end{array}\right] \quad, \quad \mathrm{u} \quad$ and $\quad \mathrm{v}, \quad$ respectively, corresponding to the positive and negative part of the vector $\mathrm{x}, \mathrm{x}=\mathrm{u}-\mathrm{v}, \quad u \geq 0 v \geq 0,1_{2 n}$ 2n-dimensional unit column vector.

The realizing process of ADGPSR as follows:

Step 1: Choose parameters

$\alpha_{\min }, \alpha_{\max }, \alpha_{0} \in\left[\alpha_{\min }, \alpha_{\max }\right]$, set $k=0$.
Then, According to the formula (X) compute $\alpha_{0}$

$$
\alpha_{0}=\frac{\left(g^{(k)^{T}}\right) g^{(k)}}{\left(g^{(k)^{T}}\right) B g^{(k)}}
$$

Step 2: Compute step

$$
\delta^{(k)}=\left(z^{(k)}-\alpha^{(k)} \nabla f\left(z^{(k)}\right)-\alpha^{(k-1)} \nabla f\left(z^{(k-1)}\right)-z^{(k)}\right.
$$

Step 3:Search $\chi^{(k)} \in[0,1]$ in the interval to a minimum $\chi^{(k)}$,and set

$$
Z^{(k+1)}=z^{(k)}+\lambda_{1}^{(k)} \delta^{(k)}+\lambda_{2}^{(k-1)} \delta^{(k-1)}
$$

Approximation $Z^{(k+1)}$ solution then the algorithm terminates, $Z^{(k+1)}$ is the reconstructed image, otherwise look for $\chi^{(k)}$.

$$
\alpha_{1}^{(k)}=\frac{\left\langle\nabla F\left(z^{(k)}\right)\right\rangle}{\left\|C^{(k)}\right\|_{2}^{2}} \alpha_{1}^{(k-1)}=\frac{\left\langle\nabla F\left(z^{(k-1)}\right)\right\rangle}{\left\|C^{(k-1)}\right\|_{2}^{2}}
$$

Where, $c^{(k)}$ is the vector $C^{(k)}=\nabla F(z)$

The parameter $\gamma^{(k)}$ is compute as follows,

$$
\gamma^{(k)}=\left(\delta^{(k)}\right)^{T} B \delta^{(k)}
$$

Where,

$$
\gamma_{1,2}{ }^{(k)}=\operatorname{mid}\left\{0, \frac{\delta^{(k)^{T}} \nabla F\left(z^{(k)}\right)}{\left(\delta^{(k)}\right)^{T} B \delta^{(k)}}, 1\right\}
$$

The parameter $\nabla F(z)$ is compute as follows,

$$
F(z)=\min c^{T} z+\frac{1}{2} z^{T} B z
$$

Where value of $z=\left\{\begin{array}{l}1 \quad \text { if } \quad z_{(j)}>0 \\ -1 \text { if } \quad z_{(j)}<0\end{array}\right.$

$j=1,2, \cdots k, z_{(k)}$ is the reconstruction vector

In the ADGPSR method, we compute the compute the gradient direction of $Z_{(k)}$ and the step length of $\delta_{1}$ and $\delta_{2}$, thus we can select the gradient direction of $Z_{(k)}$, in order to reduce the alternately search ,thus the run time is reduced.

We choose the negative direction of $Z_{(k)}$ and $Z_{(k-1)}$ firstly, if the termination is reached, the algorithm stops, otherwise, we choose the positive direction of $Z_{(k)}$ and $Z_{(k-1)}$. 


\section{EXPERIMENT RESULTS AND ANALYSIS}

\section{A Signal reconstruction Test}

In the first experiment, we set original signals length is 1024 , observation matrix is $k \times n$ gauss random matrix, $\mathrm{k}=256$ is the length of observation vector. There are 40 random \pm 1 spikes, In ADGPSR algorithm, the $\chi_{k} \equiv 1, \alpha_{\min }=10^{-30}, \alpha_{\max }=10^{30}$.tau $=0.005$.

The original signals and reconstruction signals are shown in Figure1.Experiment results show that, the MES of ADGPSR algorithm is lower than the GPSR algorithm .

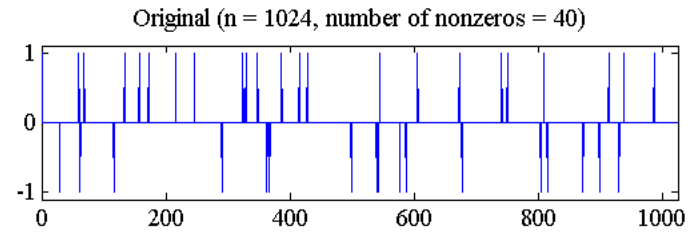

ADGPSR Algorithm ( $\mathrm{MSE}=4.34 \mathrm{e}-006)$

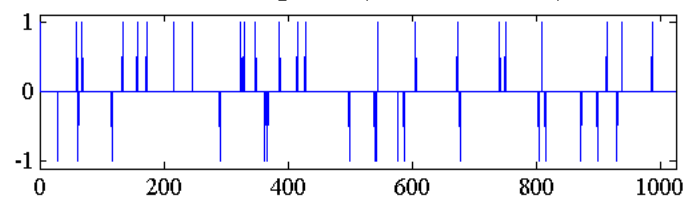

GPSR Algorithm $(\mathrm{MSE}=5.203 \mathrm{e}-006)$



Figure 1 MSE of original signals and reconstruction signals

\section{$B$ Image reconstruction Test}

We know that the image is not sparse itself, in this article, we use the wavelet transform sparse the signal. And the random Gaussian matrix as the observation matrix, the variance distribution $(0,1 / N)$. The reconstruction algorithm uses the ADGPSR(Adaptive Gradient Projection for Sparse Reconstruction).

We use the GOLDHILL and RICE image to test the efficient of ADGPSR algorithm, the image size is $256 \times 256$ and $512 \times 512$ differently, at the same time we use GPSR algorithm reconstruction image. The figure 2 shows the GOLDHILL image test results. figure 2(a) is the original GOLDHILL image, figure 2(b) is the noise GOLDHILL image with noise variance equals to 2, figure 2(c) is the ADGPSR reconstruction results from noise image, figure 2(d) is the GPSR reconstruction results from noise image. With the same input noise image the GPSR method PSNR is 25.83, the GPSR method PSNR is 22.78.

To compute the PSNR, the block first calculates the mean-squared error using the following equation formula(17),

$$
M S E=\frac{\sum_{M, N}\left[I_{1}(m, n)-I_{2}(m, n)\right]^{2}}{M \times N}
$$

We compute the PSNR with the follows formula(18),

$$
P S N R=10 \log _{10}\left(R^{2} / M S E\right)
$$

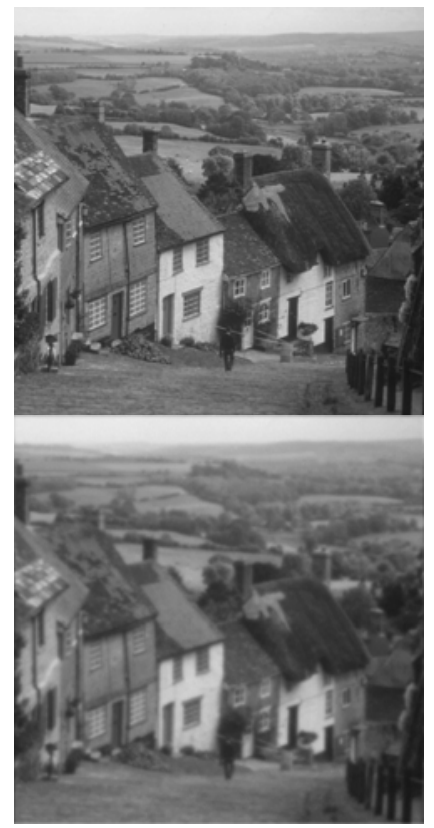

(a) GOLDHILL image

(b)noise image
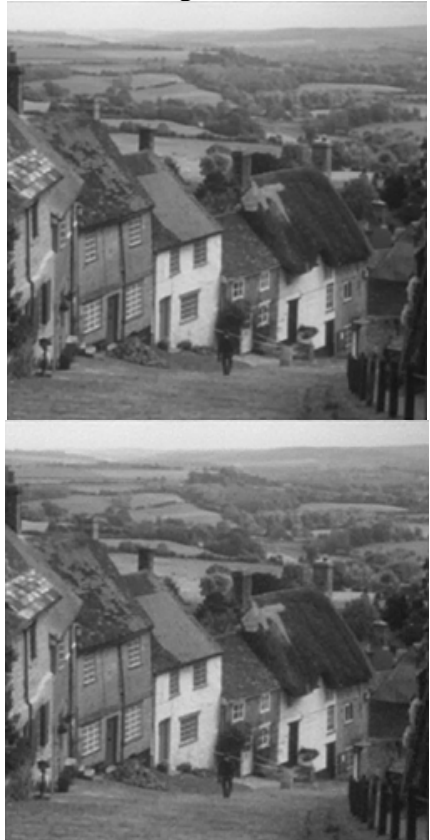

(c) ADGPSR results(PSNR=25.83) (d) GPSR results $(\mathrm{PSNR}=22.78)$

Figure 2 GOLDHILL image and reconstruction results In order to test the adaptability of the ADGPSR reconstruction algorithm, we use the RICE image to test. Figure 3(a) is the original RICE image , figure 3(b) is the 
noise RICE image with noise variance equals to 2, figure 3(c) is the ADGPSR reconstruction results from noise image, figure 3(d) is the GPSR reconstruction results from noise image. With the same input noise image the ADGPSR method PSNR is 16.37, the GPSR method PSNR is 14.07.

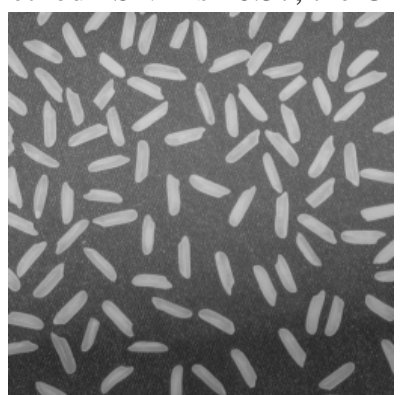

(a) RICE image

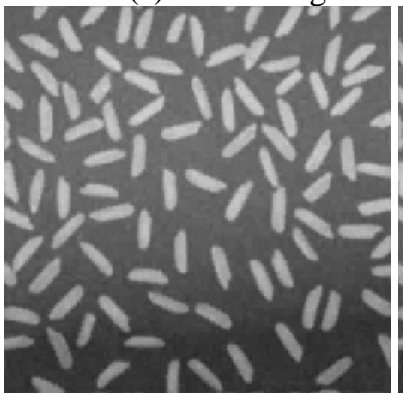

(c) ADGPSR results(PSNR=16.37) (d) GPSR results

$(\mathrm{PSNR}=14.07)$

Figure 3 RICE image and reconstruction results

\section{SUMMARY}

In this paper, ADGPSR algorithm (adaptive dual gradient projection for sparse reconstruction ) is proposed, in ADGPSR algorithm when compute the gradient projection of we compute two conjugate direction of pursuit. The signals reconstruction quality of ADGPSR is higher than GPSR algorithm. We test the ADGPSR with non-zero signals and noise image of RICE and GOLDHILL, Compared with GPSR algorithm, the reconstruction quality be enhanced. The PSNR is improved 2 3dB.

Contact Author: Liu Yanjun, E-mail: liuyanjun@ ciomp.ac.cn

\section{REFERENCES}

[1] D.Donoho,Compressed sensing[J].IEEE Trans.Inform.Theory.2006, 52 (4): 1289-1306.

[2] Candès,E.J.,Romberg,J.,Tao,T.,Signal recovery from incomplete and inaccurate measurements[J].Comm.Pure.Appl.Math.2005,59(8):1207223.

[3] Donoho D,Huo X,Uncertainty Principles and Ideal Atomic Decompositions[J].IEEE Trans Inform Theory,2001,47(7):2845-2862.
[4] S.S.Chen,D.L.Donoho,M.A.Saunders,Atomic decomposition by basis pursuit[J],SIAM Journal of Scientific Computing,1998,20(1):33-61.

[5] M.Figueiredo,R.Nowak,S.Wright,Gradient projection for sparse reconstruction:Application to compressed sensing and other inverse problems[J],IEEE Journal of Slaected Topics in Signal Processing,2007. 12,1(4):586-597.

[6] E.Candès,J.Romberg,T.Tao,Robust uncertainty principles:Exact signal reconstruction from highly incomplete frequency information[J],IEEE Trans.Inform.Theory,2006,52(2):489-509.

[7] Mun, Sungkwang. Residual reconstruction for block-based compressed sensing of video[J].Data Compression Conference Proceedings, p 183-192, 2011, Proceedings - DCC 2011: 2011 Data Compression Conference.

[8] Plumbley, Mark D.Sparse reconstruction for compressed sensing using stagewise polytope faces pursuit[J].DSP 2009: 16th International Conference on Digital Signal Processing, Proceedings, 2009, DSP 2009:16th International Conference on Digital Signal.

[9] Lei, J. Generalized reconstruction algorithm for compressed sensing[J].Computers and Electrical Engineering, v 37, n 4, p 570588, July 2011.

[10] Krishnan, Anantha Raman, Sankararaman, Swaminathan, etc.Graphbased iterative reconstruction of sparse signals for compressed sensing[J].2011 10th International Conference on Telecommunications in Modern Satellite, Cable and Broadcasting Services, TELSIKS 2011 - Proceedings of Papers, p 133-137, 2011, 2011 10th International Conference on Telecommunications in Modern Satellite, Cable and Broadcasting Services, TELSIKS 2011 Proceedings of Papers.

[11] Zhang, Y.A two-level iterative reconstruction method for compressed sensing MRI[J].Journal of Electromagnetic Waves and Applications, v 25, n 8-9, p 1081-1091, 2011.

[12] Le Montagner, Yoann, Marim, Marcio; Angelini, Elsa. etc.Numerical evaluation of sampling bounds for near-optimal reconstruction in compressed sensing[J].Proceedings - International Conference on Image Processing, ICIP, p 3073-3076, 2011, ICIP 2011: 2011 18th IEEE International Conference on Image Processing.

[13] Ni, Kangyu, Datta, Somantika; Mahanti. etc.Using reed-muller sequences as deterministic compressed sensing matrices for image reconstruction[J]. ICASSP, IEEE International Conference on Acoustics, Speech and Signal Processing - Proceedings, p 465-468, 2010, 2010 IEEE International Conference on Acoustics, Speech, and Signal Processing, ICASSP 2010 - Proceedings.

[14] Carrillo, Rafael E. Barner, Kenneth E.; Aysal. etc.Robust sampling and reconstruction methods for compressed sensing[J].ICASSP, IEEE International Conference on Acoustics, Speech and Signal Processing - Proceedings, p 2881-2884, 2009, 2009 IEEE International Conference on Acoustics, Speech, and Signal Processing Proceedings, ICASSP 2009.

[15] Tang, Jie Nett, Brian; Chen, Guang-Hong.Performance comparison between compressed sensing and statistical iterative reconstruction algorithms[J].rogress in Biomedical Optics and Imaging Proceedings of SPIE, v 7258, 2009, Medical Imaging 2009: Physics of Medical Imaging.

[16] Dixon, Anna M.R. Allstot, Emily G.; Chen, Andrew Y.ect.Compressed sensing reconstruction: Comparative study with applications to ECG bio-signals[J].Proceedings - IEEE International Symposium on Circuits and Systems, p 805-808, 2011, 2011 IEEE International Symposium of Circuits and Systems, ISCAS 2011. 